\title{
Dermatopatias em equinos no estado de Santa Catarina
}

\section{Dermatopathies in horses in the state of Santa Catarina}

\author{
Aline Tamye Izutani Aragão1 ${ }^{1}$ Jacqueline Raiter ${ }^{2}$, Luis Fagner da Silva Machado³, José Eduardo Basilio de Oliveira \\ Gneiding ${ }^{3,4}$, Beatriz Gneiding ${ }^{4}$, Joelma Lucioli ${ }^{*}$ \\ ${ }^{1}$ Clínica Médica e Cirúrgica de Equinos, Brasília, DF, Brasil \\ ${ }_{2}^{2}$ Programa de Residência Multiprofissional em Saúde Animal e Coletiva (Patologia Veterinária), Universidade Federal do Rio Grande do \\ Sul (UFRGS), Porto Alegre, RS, Brasil \\ ${ }^{3}$ Departamento de Medicina Veterinária, Universidade Regional de Blumenau (FURB), Blumenau, SC, Brasil \\ ${ }^{4}$ Programa de Pós- Graduação em Ciencia Animal, Pontificia Universidade Católica do Paraná (PUCPR), Curitiba, PR, Brasil
}

\section{Resumo}

Este trabalho consiste em um estudo retrospectivo de doenças da pele de equinos diagnosticadas no Laboratório de Patologia Veterinária (LAPAVET/ HEV/FURB), no período de três anos (2013-2015). Para determinar a prevalência de dermatopatias em equinos, foram compilados e apontados os seguintes dados: diagnóstico histopatológico, sexo, idade, raça e localização anatômica da lesão. As dermatopatias mais prevalentes foram: sarcóide e habronemose (15/62 cada - 24,2\%), localizados frequentemente em membros torácicos e pélvicos; hipersensibilidade à picada de inseto (12/62 - 19,35\%) observada comumente na região da cauda; e carcinoma de células escamosas (5/62 - 8,1\%) em região urogenital. Demais lesões cutâneas representaram $24,2 \%$ de todos os casos estudados. Dentre as raças, a mais prevalente foi a Crioulo (21/62 - 33,9\%), e quanto à idade, os animais com idade de 6-14 anos (19/62 - 30,6\%) foram os mais afetados. A maioria das dermatopatias observadas nesse estudo não resultaram na morte do animal, embora tenham causado alterações estéticas, comportamentais e de performance, resultando na incapacidade de participação em atividades esportivas e/ou de trabalho.

Palavras-chave: Dermatologia. Patologia. Afecções da pele. Tegumento.

\begin{abstract}
This survey consists of a retrospective study of equine skin diseases diagnosed in the Laboratory of Veterinary Pathology (LAPAVET/HEV/FURB) in a three years period (2013-2015). To determine the prevalence of dermatopathies in horses, the following data were compiled and identified: histopathological diagnosis, sex, age, race and anatomical location of the lesion. The most prevalent dermatopathies were: sarcoid and habronemosis
\end{abstract}


(15/62 each - 24.2\%), often located in the thoracic and pelvic limbs; hypersensitivity to insect bite (12/62 $19.35 \%)$ commonly observed in tail region; and squamous cell carcinoma (5/62 - 8.1\%) in the urogenital region. Other cutaneous lesions represented $24.2 \%$ of all the cases studied. Among the breeds, the most prevalent was Crioulo (21/62 - 33.9\%), and regarding age, 6-14 years old (19/62 - 30.6\%) animals were the most affected Most of the skin diseases observed in this study did not result in the death of the animal although they have caused aesthetic, behavioral and performance changes, resulting in the inability to participate in sports and/or work activities.

Keywords: Dermatology. Pathology. Skin disorders. Tegument.

\section{Introdução}

Lesões cutâneas estão entre as queixas clínicas mais frequentes em diferentes animais, sendo os equinos a terceira espécie mais acometida por dermatopatias (Scott e Miller Jr., 2003). Sua prevalência é bem conhecida nos Estados Unidos e Europa (Pascoe e Knottenbelt, 1999; Fadock, 2003; Lloyd et al., 2003; Scott e Miller, 2003; Mueller, 2005; Meierhenry, 2006; Ludgate et al., 2010; Ireland et al., 2012; Knowles et al., 2015), entretanto quase nada se sabe sobre a prevalência destas em equinos no Brasil (Ramos et al., 2007; Rissi et al., 2008; Souza et al., 2011; Pessoa et al., 2014, Assis-Brasil et al., 2015), país detentor do terceiro maior rebanho equídeo do mundo (Brasil, 2016), e principalmente no estado de Santa Catarina, o que ressalta a importância do conhecimento e compreensão das patologias do tegumento nessa espécie.

Afecções dermatológicas possuem sinais clínicos diversos, como alopecia, ulcerações e prurido, e podem ocasionar processos que geram desconforto no animal, levando-os a mudanças de comportamento, diminuição da concentração e redução da performance daqueles utilizados para qualquer modalidade de esporte e/ou trabalho (Meierhenry, 2006).

Há uma grande quantidade de patologias de pele em equídeos mostrando similaridade clínica. Por este motivo, o diagnóstico da doença dermatológica requer uso de técnicas especializadas, dentre elas o raspado de pele, citologia, tricograma, cultura, antibiograma e histopatologia (Londoño et al., 2014).

Devido à importância socioeconômica da espécie e à necessidade de maior compreensão das dermatopatias que acometem os equinos no estado de Santa Catarina, a proposta desse estudo foi determinar a prevalência das afecções cutâneas diagnosticadas no Laboratório de Patologia Veterinária (LAPAVET/HEV) da Universidade Regional de Blumenau (FURB).

\section{Material e métodos}

Foi realizado estudo retrospectivo de casos associados a lesões cutâneas em equídeos, tendo como base os arquivos do Laboratório de Patologia Veterinária do Hospital Escola Veterinário da Universidade Regional de Blumenau (LAPAVet/ HEV/FURB) no período de três anos (2013-2015). Foram compiladas e apontadas informações como diagnóstico histopatológico, sexo, idade, raça e localização anatômica da lesão. Quanto à idade, os animais foram agrupados por faixa etária, considerando: jovens ( 1 a 5 anos), adultos ( 6 a 14 anos), senis (15 anos ou mais) e não informado. Em relação à localização anatômica, consideraram-se: membros, tronco, pescoço, cabeça, cauda, região urogenital e lesões multifocais. Alguns protocolos não continham informações a respeito da localização das lesões, dessa forma foram classificadas apenas como "pele".

Os dados foram organizados em planilhas por meio do software Microsoft Excel ${ }^{\circledR}$ versão 2013 e analisados no software Epi Info ${ }^{\circledR}$ versão 7.0. Resultados das análises foram disponibilizados na forma de tabelas, evidenciando frequências absolutas e relativas (proporções), sendo adotado o nível de significância de $\mathrm{p} \geq 0,05$.

\section{Resultados e discussão}

Durante o período de três anos (2013-2015), o LAPAVet/HEV/FURB recebeu um total de 62 amostras de pele de equídeos para realização de exames histopatológicos. A partir do levantamento de fichas 
preenchidas com os respectivos dados, foi possível realizar um perfil dos animais (Tabela 1). Quanto ao sexo, observou-se que os machos foram os mais acometidos, representando 53,3\% (33/62) do total, corroborando os achados de Sánchez-Casanova et al. (2014), que observaram um maior comprometimento dos machos em relação às fêmeas, e diferindo dos achados de Souza et al. (2011), Bianchi (2013) e AssisBrasil et al. (2015), que relatam maior prevalência de dermatopatias em fêmeas. Nenhuma das patologias cutâneas diagnosticadas no presente estudo possui predileção sexual descrita em literatura.

Tabela 1 - Dados de prevalência, por categoria, dos equinos diagnosticados com afecções dermatológicas pelo LAPAVet/ HEV/FURB

\begin{tabular}{lcc}
\hline \multicolumn{1}{c}{ Caracteristicas } & $\mathbf{N}$ & IC (95\%) \\
\hline Sexo & & \\
Fêmeas & $26(41,9 \%)$ & $(29,65-54,22)$ \\
Machos & $33(53,2 \%)$ & $(40,81-65,65)$ \\
Não informado & $3(4,8 \%)$ & $(0-10,18)$ \\
Idade & & \\
1 a 5 & $14(22,6 \%)$ & $(12,17-32,99)$ \\
6 a 14 & $19(30,6 \%)$ & $(19,17-42,12)$ \\
$>15$ & $9(14,5 \%)$ & $(5,75-23,28)$ \\
Não informado & $20(32,3 \%)$ & $(20,62-43,89)$ \\
Raça & & \\
Crioulo & $21(33,9 \%)$ & $(22,09-45,65)$ \\
Mangalarga & $8(12,9 \%)$ & $(4,56-21,25)$ \\
Brasileiro de hipismo & $5(8,1 \%)$ & $(1,29-14,84)$ \\
Quarto de milha & $2(3,2 \%)$ & $(0-7,62)$ \\
Muar & $1(1,6 \%)$ & $(0-4,75)$ \\
Appaloosa & $1(1,6 \%)$ & $(0-4,75)$ \\
SRD & $13(21 \%)$ & $(10,83-31,10)$ \\
Não informado & $11(17,7 \%)$ & $(8,23-27,25)$ \\
\hline
\end{tabular}

Nota: $\mathrm{N}$ - amostra; IC - intervalo de confiança.

A idade foi informada em $67,6 \%$ dos casos atendidos, observando-se acometimento em animais entre 1 e 24 anos (Tabela 1). Entretanto, a faixa etária dos 6 a 14 anos apresentou 19 (30,6\%) animais acometidos, corroborando com os achados de Bianchi (2013), que constatou maior prevalência de lesões nesta mesma faixa etária. Já Pierezan et al. (2009) e Souza et al. (2011) observaram prevalência maior em animais entre um e cinco anos. Talvez este achado esteja relacionado a não comunicação da idade quando solicitado o exame em aproximadamente $1 / 3$ dos casos $(32,3 \%)$ registrados.

As raças Crioula e Mangalarga, por sua versatilidade, são amplamente utilizadas para trabalho, lazer, esporte e prática de cavalgada, sendo predominantes no sul do país (Brasil, 2015), o que justifica o número maior de exemplares dessas raças no presente estudo. Resultados semelhantes foram observados por Souza et al. (2011), Bianchi (2013) e Assis-Brasil et al. (2015) em trabalhos similares realizados no Rio Grande do Sul.

Dentre as lesões diagnosticadas (Tabela 2), as de origem neoplásica de maior prevalência foram o sarcóide equino, com $24,2 \%$ (15/62), seguido pelo carcinoma de células escamosas, com 8,1\% (5/62) (Figuras 1 e 2). De todas as neoplasias cutâneas, o sarcóide equino é a mais comum (Scott e Miller, 2003; Souza et al., 2011; Bianchi, 2013; Pessoa et al., 2014; Sánchez-Casanova et al., 2014; Assis-Brasil et al., 2015), e quando comparado com outras neoplasias (Souza et al., 2011; Bianchi, 2013), geralmente acomete animais mais jovens. Um dos principais fatores etiológicos do sarcóide equino é a exposição ao papilomavirus bovino (BPV) do tipo 1 e 2 , que está relacionado ao contato direto com bovinos.

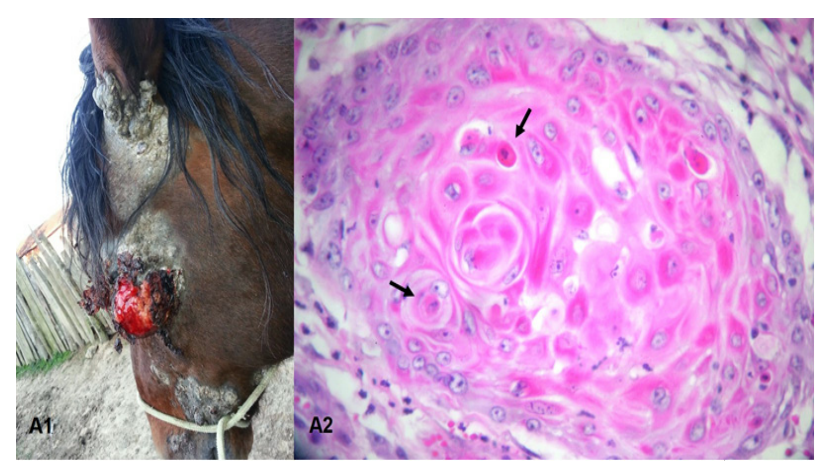

Nota: A1 - Aumento de volume localizado em região periocular esquerda. A2 - Corte histológico demonstrando células epiteliais com diferentes graus de diferenciação, algumas se diferenciando em queratinócitos (seta). HE, obj. 40x.

Figura 1 - Análise macroscópica e histológica de carcinoma de células escamosas. 


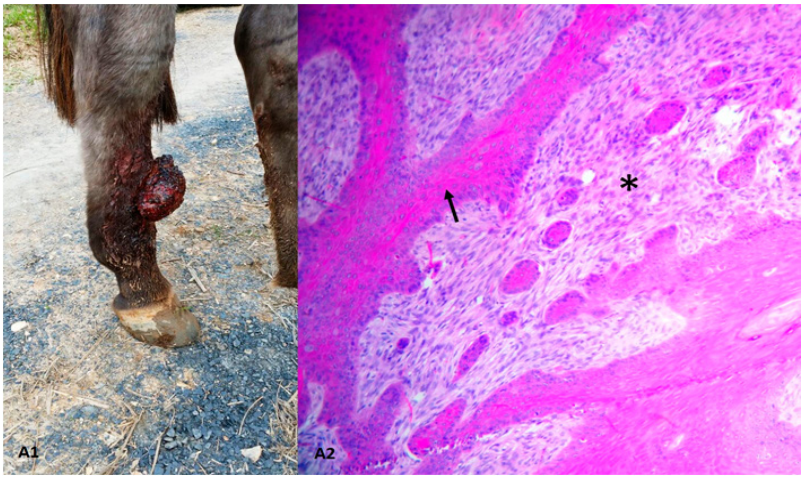

Nota: A1 - Aumento de volume localizado em região de metatarso.A2 - Corte histológico demonstrando intensa proliferação fibroblástica (asterisco) com extensas projeções epiteoliomatosas (seta) em direçao a derme. HE, obj. 40x.

Figura 2 - Análise macroscópica e histológica de sarcoide equino.

Tabela 2 - Afecções dermatológicas de equinos diagnosticadas pelo LAPAVet/HEV/FURB

\begin{tabular}{lcc}
\hline \multicolumn{1}{c}{ Diagnóstico } & N & Prevalência \\
\hline Habronemose cutânea & 15 & $24,2 \%$ \\
Sarcóide & 15 & $24,2 \%$ \\
Hipersensibilidade cutânea à picada & 12 & $19,35 \%$ \\
de inseto & & \\
Carcinoma de células escamosas & 5 & $8,1 \%$ \\
Tecido de granulação & 4 & $6,45 \%$ \\
Papiloma & 2 & $3,22 \%$ \\
Melanoma & 2 & $3,22 \%$ \\
Pitiose & 1 & $1,61 \%$ \\
Granuloma & 1 & $1,61 \%$ \\
Adenoma de glândula sebácea & 1 & $1,61 \%$ \\
Hiperplasia fibroepitelial & 1 & $1,61 \%$ \\
Hemangioma & 1 & $1,61 \%$ \\
Dermatite atópica & 1 & $1,61 \%$ \\
Piodermite superficial com & 1 & $1,61 \%$ \\
dermatite perivascular superficial & & \\
\hline Total & 62 & $100 \%$ \\
\hline
\end{tabular}

Nota: N - amostra; IC - intervalo de confiança.

Para o sarcóide equino, a raça mais prevalente foi a Crioula, com 33,3\% dos casos diagnosticados (Tabela 3 ), o que corrobora com os estudos de Souza et al. (2011), Bianchi (2013) e Assis-Brasil et al. (2015).
Animais de sela, como o Crioulo e o Mangalarga, quando comparados com animais de corrida, são mais predispostos ao desenvolvimento de sarcóide equino devido ao maior contato com bovinos (Scott e Miller, 2003; Assis-Brasil et al., 2015).

Assim como descrito na literatura, o carcinoma de células escamosas (CCE) é a segunda neoplasia mais frequente em equinos (Scott e Miller, 2003), em concordância ao presente estudo, onde sua prevalência representou 8,1\% (5/62). Pierezan et al. (2009), Souza et al. (2011), Bianchi (2013), Pessoa et al. (2014) e Assis-Brasil et al. (2015), ao estudarem lesões de tegumento em equinos, obtiveram resultados que se assemelham aos dados encontrados neste estudo. Baccarin et al. (2011), contudo, relataram o CCE como sendo a principal neoplasia cutânea encontrada. Uma das causas etiológicas do CCE é a exposição excessiva a raios UV, além do aumento da predisposição em animais de peles despigmentadas, o que pode se correlacionar a equinos de trabalho que sofreram exposição solar constante e por longos períodos (Baccarin et al., 2011; Souza et al., 2011; Bianchi, 2013). A raça Appallosa possui predisposição genética para o desenvolvimento de neoplasias tegumentares por possuir despigmentação cutânea, queé característica em várias pelagens da raça (Ramos et al., 2007).

Entre as dermatopatologias não neoplásicas, a de maior prevalência foi a habronemose, com $24,2 \%$ (15/62), seguida pela hipersensibilidade cutânea, com 19,35\% (12/62), conforme demonstrado na Tabela 2. A raça Crioula representou 46,6\% (7/15) e a Mangalarga 26,6\% (4/15) do total de diagnósticos para habronemose, sendo estas as raças de maior frequência (Tabela 3).

A habronemose cutânea está diretamente relacionada a climas tropicais e subtropicais, onde ocorre a maior proliferação de insetos, principalmente entre os meses de janeiro e maio na Região Sul do país (Bianchi, 2013; Assis-Brasil et al., 2015). A sazonalidade também está diretamente relacionada com as estações chuvosas, nas quais ocorre aumento da população de Stomoxys calcitrans e Musca domestica (Pessoa et al., 2014), sendo Santa Catarina um estado conhecido pelo alto índice pluviométrico durante o ano. Sabe-se que equinos das raças Crioula e Mangalarga são amplamente utilizados no estado para diversas 
funções de trabalho e lazer, o que torna maior o risco de ferimentos de pele, sendo a habronemose cutânea (Figura 3) uma enfermidade secundária a lesões de continuidade do tegumento.

Além disto, animais criados de maneira extensiva ou em locais com higiene insuficiente podem apresentar maior risco no desenvolvimento da doença, devido a maior exposição a insetos e a maior proliferação dos mesmos, o que possivelmente explica a elevada prevalência dessas duas raças (Tabela 3) em nosso estudo, diferindo dos achados de Bianchi (2013), Pessoa et al. (2014) e AssisBrasil et al. (2015), que relataram uma prevalência menor no estado do Rio Grande do Sul. Em adição, os mesmos autores obtiveram mais casos de pitiose do que de habronemose cutânea, o que pode ser explicado pelo fato de a economia do estado integrar a agricultura com a pecuária e possuir grandes extensões de plantação de arroz irrigado, o que submete os animais a ficarem em águas estagnadas durante longos intervalos de tempo (MarcolongoPereira et al., 2012).

Quanto à hipersensibilidade cutânea, esta pode ter diversas etiologias, entretanto a dermatite alérgica à picada de insetos (Figura 4) ou hipersensibilidade a Culicoides sp. é a mais comum (Scott e Miller, 2003), e para sua ocorrência basta que haja condições ambientais para a proliferação de insetos. No presente estudo, conforme demonstrado na Tabela 3, as raças mais acometidas foram a Brasileiro de Hipismo, com 33,3\% (4/12), seguida da Crioula e SRD, com 25\% (3/12) cada. 0 estudo de Pessoa et al. (2014) apresentou prevalência menor quando comparado a este trabalho, o que pode estar associado a fatores ambientais desfavoráveis à proliferação desses insetos no semiárido.

Tabela 3 - Associação entre diagnóstico e raça em equinos com afecções dermatológicas diagnosticadas no LAPAVet/HEV/FURB

\begin{tabular}{lcccccccc}
\hline & \multicolumn{7}{c}{ Raças } \\
\cline { 2 - 9 } Patologia & Crioulo & Mangalarga & $\begin{array}{c}\text { Quarto de } \\
\text { milha }\end{array}$ & Appaloosa & $\begin{array}{c}\text { Brasileiro } \\
\text { de hipismo }\end{array}$ & Muar & SRD & NI \\
Habronemose cutânea & $7(33,3 \%)$ & $4(50 \%)$ & $1(50 \%)$ & - & - & - & $1(7,7 \%)$ & $2(18,1 \%)$ \\
Sarcóide & $7(33,3 \%)$ & $1(12,5 \%)$ & - & - & - & - & $3(23,05 \%)$ & $4(36,4 \%)$ \\
Hipersensibilidade cutânea à & $3(14,2 \%)$ & $1(12,5 \%)$ & $1(50 \%)$ & - & $4(80 \%)$ & - & $3(23,05 \%)$ & - \\
picada de inseto & & & & & & & & \\
Carcinoma de células escamosas & $1(4,8 \%)$ & - & - & $1(100 \%)$ & - & - & $1(7,7 \%)$ & $2(18,2 \%)$ \\
Tecido de granulação & $2(9,6 \%)$ & $1(12,5 \%)$ & - & - & - & - & $1(7,7 \%)$ & - \\
Papiloma & $1(4,8 \%)$ & - & - & - & - & - & $1(7,7 \%)$ & - \\
Melanoma & - & - & - & - & - & - & - & $2(18,2 \%)$ \\
Pitiose & - & - & - & - & - & $1(100 \%)$ & - & - \\
Granuloma & - & - & - & - & - & - & - & $1(9,1 \%)$ \\
Adenoma de glândula sebácea & - & - & - & - & - & - & $1(7,7 \%)$ & - \\
Hiperplasia fibroepitelial & - & - & - & - & - & - & $1(7,7 \%)$ & - \\
Hemangioma & - & $1(12,5 \%)$ & - & - & - & - & - & - \\
Dermatite atópica & - & - & - & - & $1(20 \%)$ & - & - & - \\
Piodermite superficial com & - & - & - & - & - & - & $1(7,7 \%)$ & - \\
dermatite perivascular superficial & & & & & & & & \\
\hline TOTAL & $21(100 \%)$ & $8(100 \%)$ & $2(100 \%)$ & $1(100 \%)$ & $5(100 \%)$ & $1(100 \%)$ & $13(100 \%)$ & $11(100 \%)$ \\
\hline
\end{tabular}

Nota: SRD - sem raça definida; NI - raça não informada. 


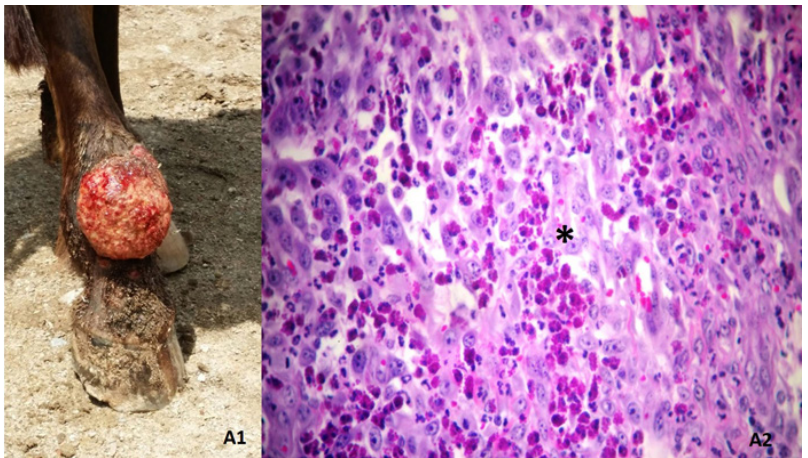

Nota: A1 - Aumento de volume localizado em região distal de membro pélvico (boleto). A2 - Corte histológico demonstrando intenso infiltrado inflamatório com predomínio de eosinófilos (*). HE, obj. 40x.

Figura 3 - Análise macroscópica e histológica de habronemose.

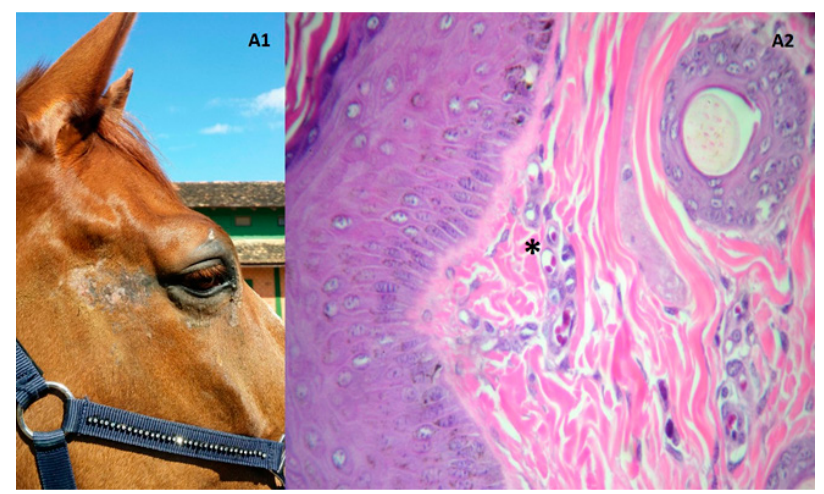

Nota: A1 - Área focalmente extensa de alopecia próximo a região ocular. A2 - Corte histológico demonstrando infiltrado perivascular mononuclear leve $\left(^{*}\right)$ em derme superficial. HE, obj. 40x.

Figura 4 -Análise macroscópica e histológica de hipersensibilidade cutânea por picada de insetos.

Alguns estudos comprovam a predisposição genética ao desenvolvimento da doença em equinos das raças Fresian, Quarto de Milha, Puro Sangue Inglês, Puro Sangue Árabe e pônei Shetland Holandês (Schaffartzik et al., 2012; Peeters et al., 2015; Shrestha et al., 2015), entretanto, nenhum estudo foi realizado no país com o intuito de verificar a existência de predisposição racial. Sabese que a raça Brasileiro de Hipismo possui em sua formação racial a genética de equinos Puro Sangue Inglês e Anglo-Árabe (Dias et al., 2000), o que pode justificar a sua predisposição à patologia.
Quanto à localização anatômica das lesões, 27 $(43,5 \%)$ dos formulários não possuíam informação a respeito da região anatômica acometida, estando apenas indicado "pele" (Tabela 4). A partir das informações obtidas, os membros pélvicos e torácicos foram os mais acometidos, com 10 casos $(16,1 \%)$, seguidos de cabeça e urogenital, ambos com seis casos $(9,6 \%)$ (Tabela 4). A habronemose se apresentou principalmente em membros, corroborando com os estudos de Bianchi (2013), porém contrariando os de Pessoa et al. (2014), que observaram maior acometimento da região da cabeça. Estudos relatam que as regiões mais acometidas pela habronemose são membros e face (Assis-Brasil et al., 2015), o que pode ser explicado por estas regiões anatômicas estarem mais propensas a traumas diretos de tegumento, associado ao fato de a cabeça possuir mais junções mucocutâneas quando comparada a outras regiões corpóreas.

Assim como a habronemose, o sarcóide equino acometeu principalmente a região dos membros, representando $40 \%$ (4/10) de toda a frequência (Tabela 4), o mesmo encontrado por Bianchi (2013) e Pessoa et al. (2014). Os membros são muito propensos a traumatismos, sendo que o sarcóide inicia seu desenvolvimento secundário a uma lesão cutânea (Bergvall, 2013), o que justifica a maior frequência nesta região. Em relação à hipersensibilidade cutânea, os locais mais acometidos foram a cauda e o pescoço, representando $100 \%(6 / 6)$ das lesões em cauda e $75 \%$ (3/4) de todas lesões em pescoço, o que corrobora com os achados de Schaffartzik et al. (2012) e Fadok (2013); Scott e Miller (2003), entretanto, relatam lesões multifocais.

Como encontrado por Bianchi (2013), o carcinoma de células escamosas se mostrou presente principalmente na região urogenital, onde a neoplasia representou $40 \%(2 / 5)$ de todas as lesões que acometeram esta área (Tabela 4), sendo todos os animais acometidos do sexo masculino. Pessoa et al. (2014) e Baccarin et al. (2011), porém, obtiveram maior frequência na região da cabeça. Estudos relatam que a ação carcinogênica do smégma de indivíduos machos pode ser um fator etiológico para o desenvolvimento desta neoplasia na região urogenital (Van Den Top et al., 2010). 
Tabela 4 - Associação entre o diagnóstico e a localização anatômica das afecções dermatológicas diagnosticadas no LAPAVet/HEV/FURB

\begin{tabular}{lccccccccc}
\hline & \multicolumn{7}{c}{ Localização anatômica } \\
\cline { 2 - 8 } Diagnóstico & Cabeça & Cauda & Face & Membro & Multifocal & Pele & Pescoço & Tronco & Urogenital \\
Habronemose cutânea & 1 & - & - & 3 & 1 & 9 & - & - & 1 \\
Sarcóide & 1 & - & - & 4 & 1 & 8 & - & 1 & - \\
Hipersensibilidade cutânea à picada de inseto & 1 & 6 & - & 1 & 2 & 3 & 3 & - & - \\
Carcinoma de células escamosas & 1 & - & - & - & - & 2 & - & - & 2 \\
Tecido de granulação & - & - & - & 2 & - & 2 & - & - & - \\
Papiloma & 1 & - & - & - & - & 1 & - & - & - \\
Melanoma & - & - & - & - & - & - & - & - & 1 \\
Pitiose & - & - & 1 & - & - & - & - & - & - \\
Granuloma & - & - & - & - & - & 1 & - & - & - \\
Adenoma de glândula sebácea & 1 & - & - & - & - & - & - & - & - \\
Hiperplasia fibroepitelial & - & - & - & - & - & - & - & - & 1 \\
Hemangioma & - & - & - & - & - & - & 1 & - & - \\
Dermatite atópica & - & - & - & - & 1 & - & - & - & - \\
Piodermite superficial com dermatite & - & - & - & - & - & 1 & - & - & - \\
perivascular superficial & & & & & & & & & \\
\hline TOTAL & 6 & 6 & 1 & 10 & 5 & 27 & 4 & 1 & 5 \\
\hline
\end{tabular}

Constatou-se, neste estudo, que a maior dificuldade durante o processamento dos dados foi a falta de informações a respeito do perfil dos animais. A idade não foi informada em 32,2\% dos casos, assim como a raça $(17,7 \%)$. Quanto à localização anatômica, o principal achado foi para "pele" (27/65), que equivale à ausência de informação a respeito da região anatômica exata. A falta dessas informações impossibilitou que a pesquisa fosse mais completa.

\section{Conclusão}

Com base nos achados deste estudo, pode-se concluir que a habronemose, o sarcóide equino e a hipersensibilidade cutânea à picada de inseto são as patologias que mais comumente afetam a pele de equinos no estado de Santa Catarina. Observa-se, ainda, que as afecções cutâneas, de maneira geral, acometem machos, com predileção por aqueles com idade entre 6 e 14 anos.

A raça Crioula demonstrou ser a mais acometida frequentemente. Quanto à localização anatômica, habronemose e sarcóide foram mais frequentes em membros anteriores e posteriores, enquanto a hipersensibilidade cutânea à picada de inseto foi mais frequente em região de cauda.

A principal dificuldade observada durante a análise dos dados foi a falta de informações a respeito dos animais, o que pode ter interferido diretamente nos resultados. Cabe salientar que o preenchimento por completo e de forma correta de fichas clínicas e requisições de exame são extremamente importantes para a interpretação dos achados laboratoriais.

\section{Referências}

Balarin Assis-Brasil ND, Marcolongo-Pereira C, Stigger AL, Fissl L, Santos BL, Coelho ANB, et al. Equine dermatopathies in southern Brazil: a study of 710 cases. Cienc Rural. 2015;45(3):519-24.

Baccarin RYA, Silva LCLC, Belli CB, Fernandes WR, Zoppa ALV. Ocorrência de neoplasias em 15 anos de atendimento hospitalar de equídeos. Braz J Vet Res Anim Sci. 2011;48(6):439-45. 
Bianchi MV. Dermatopatias diagnosticadas em equinos no setor de patologia veterinária da UFRGS de 2000 a 2013 [monografia]. Porto Alegre: Universidade Federal do Rio Grande do Sul; 2013. 24 p.

Brasil. Ministério da Agricultura, Pecuária e Abastecimento. Revisão do estudo do complexo do agronegócio do cavalo. Brasília: MAPA; 2016. 54 p.

Dias IMG, Bergmann JAG, Rezende ACC, Castro GHF. Formação e estrutura populacional do equino Brasileiro de Hipismo. Arq Bras Med Vet Zootec. 2000;52(6):647-54.

Fadok VA. Update on equine allergies. Vet Clin North Am Equine Pract. 2013;29(3):541-50.

Ireland JL, Clegg PD, McGowan CM, McKane SA, Chandler $\mathrm{KJ}$, Pinchbeck GL. Disease prevalence in geriatric horses in the United Kingdom: veterinary clinical assessment of 200 cases. Equine Vet J. 2012;44(1):101-6.

Lloyd DH, Littlewood JD, Craig JM, Thomsett LR. Practical Equine Dermatology. Cap 5. Oxford: Blackwell Science Ltd; 2003, p. 63 - 99.

Ludgate MW, Fullen DR, Lee J, Rees R, Sabel MS, Wong SL, et al. Animal-type melanoma: a clinical and histopathological study of 22 cases from a single institution. Br J Dermatol. 2010;162(1):129-36.

Londoño ICC, San Miguel GP, Martínez GR, Arias RS. Estudio microscópico de dermatopatías en equinos de la sabana de Bogotá, Colombia. Rev Med Vet (Bogota). 2014;(27):11-20.

Marcolongo-Pereira C, Sallis ESV, Raffi MB, Pereira DIB, Hinnah FL, Coelho ACB, et al. Epidemiologia da pitiose equina na Região Sul do Rio Grande do Sul. Pesq Vet Bras. 2012;32(9):865-8.

Meierhenry B. Equine Dermatology - Everything you wanted to know (and more) about skin. CEH Horse Report. 2006;24(1):1-12.

Mueller RS. Dermatology for the Equine Practitioner. Jackson: Teton NewMedia; 2005. 110 p.

Pascoe RR, Knottenbelt DC. Manual of Equine Dermatology. Philadelphia: W.B. Saunders; 1999. 290 p.
Peeters LM, Janssens S, Brebels M, Buys N. Genetic parameters and estimated breeding values of insect bite hypersensitivity in Belgian Warmblood horses. Vet J. 2015;206(3):420-2.

Pessoa AFA, Pessoa CRM, Neto EGM, Dantas AFM, RietCorrea F. Doenças de pele em equídeos no semiárido brasileiro. Pesq Vet Bras. 2014;34(8):743-8.

Pierezan F, Rissi DR, Rech RR, Fighera RA, Brum JS, Barros CSL. Achados de necropsia relacionados com a morte de 335 equinos: 1968-2007. Pesq Vet Bras. 2009;29(3):275-80.

Ramos AT, Norte DM, Elias F, Fernandes CG. Carcinoma de células escamosas em bovinos, ovinos e equinos: estudo de 50 casos no sul do Rio Grande do Sul. Braz J Vet Res Anim Sci. 2007;44:5-13.

Rissi DR, Fighera RA, Irigoyen LF, De Lacorte FD, Barros CSL. Melanoma maligno anaplásico em um equino. Cienc Rural. 2008;38(7):2072-5.

Sánchez-Casanova RE, Masri-Daba M, Alondo-Díaz MA, Méndez-Bernal A, Hernández-Gil M, Fernando-Martínez JA. Prevalence of cutaneous pathological conditions and factors associated with the presence of skin wounds in working equids in tropical regions of Veracruz, Mexico. Trop Anim Health Prod. 2014;46(3):555-61.

Schaffartzik A, Hamza E, Janda J, Crameri R, Marti E, Rhyner C. Equine insect bite hypersensitivity: what do we know? Vet Immunol Immunopathol. 2012;147(3-4): 113-26.

Scott DW, Miller WH. Equine Dermatology. St Louis: Saunders; 2003.

Shrestha M, Eriksson S, Schurink A, Andersson LS, Sundquist M, Frey R et al. Genome-Wide Association Study of Insect Bite Hypersensitivity in Swedish-Born Icelandic Horses. J Hered. 2015;106(4):366-74.

Souza TM, Brum JS, Fighera RA, Brass KE, Barros CSL. Prevalência dos tumores cutâneos de equinos diagnosticados no Laboratório de Patologia Veterinária da Universidade Federal de Santa Maria, Rio Grande do Sul. Pesq Vet Bras. 2011;31(5):379-82. 
Van Den Top JG, Ensink JM, Gröne A, Klein WR, Barneveld A, Van Weeren PR. Penile and preputial tumours in the horse: literature review and proposal of a standardised approach. Equine Vet J. 2010;42(8):746-57. 